\title{
International Journal of Analysis and Applications
}

\section{Riemann-Liouville Fractional Versions of Hadamard inequality for Strongly $m$-Convex Functions}

\author{
Ghulam Farid $^{1}$, Saira Bano Akbar ${ }^{2}$, Laxmi Rathour ${ }^{3}$, Lakshmi Narayan Mishra ${ }^{4}$, \\ Vishnu Narayan Mishra ${ }^{5, *}$ \\ ${ }^{1}$ Department of Mathematics, COMSATS University Islamabad, Attock Campus, Pakistan \\ ${ }^{2}$ Department of Mathematics, COMSATS University Islamabad, Lahore Campus, Pakistan \\ ${ }^{3}$ Ward Number - 16, Bhagatbandh, Anuppur 484 224, Madhya Pradesh, India \\ ${ }^{4}$ Department of Mathematics, School of Advanced Sciences, Vellore Institute of Technology (VIT) \\ University, Vellore 632 014, Tamil Nadu, India \\ ${ }^{5}$ Department of Mathematics, Indira Gandhi National Tribal University, Lalpur, Amarkantak, \\ Anuppur, Madhya Pradesh 484 887, India \\ *Corresponding author: vishnunarayanmishra@gmail.com, vnm@igntu.ac.in

\begin{abstract}
This paper deals with Hadamard inequalities for strongly $m$-convex functions via RiemannLiouville fractional integrals. These inequalities provide refinements of well known fractional integral inequalities for convex functions. Further, by applying an identity error estimations are obtained and compared with already known error estimations.
\end{abstract}

1. Introduction

First, we will give some definitions and well known results which are needful and connected with the findings of this paper.

Definition 1.1. [8] A function $f: I \rightarrow \mathbb{R}$ will be called convex if

$$
f(t x+(1-t) y) \leq t f(x)+(1-t) f(y)
$$

Received: Jun. 7, 2021.

2010 Mathematics Subject Classification. 26A51, 26A33, 33E12.

Key words and phrases. $m$-convex function; strongly $m$-convex function; Hadamard inequality; Riemann-Liouville fractional integrals. 
$\forall x, y \in I=[x, y]$ and $t \in[0,1]$. The function $f$ will be strictly convex if

$$
f(x t+(1-t) y)<t f(x)+(1-t) f(y)
$$

holds $\forall x \neq y \in I$ and $t \in(0,1)$.

Definition 1.2. [7] Let $(\mathbb{X},\|\|$.$) be a normed space. A function f: E \subset \mathbb{X} \rightarrow \mathbb{R}$ will be called strongly convex function with modulus $C$ if

$$
f(x t+(1-t) y) \leq t f(x)+(1-t) f(y)-C t(1-t)\|y-x\|^{2}
$$

holds $\forall x, y \in E \subseteq \mathbb{X}, t \in[0,1]$.

Definition 1.3. [12] A function $f:[0, b] \rightarrow \mathbb{R}, b>0$ will be called m-convex function if

$$
f(t x+m(1-t) y) \leq t f(x)+m(1-t) f(y)
$$

holds $\forall x, y \in[0, b]$ and $t, m \in[0,1]$.

Definition 1.4. [6] A function $f:[0, b] \rightarrow \mathbb{R}$ will be called strongly m-convex function if

$$
f(t x+m(1-t) y) \leq t f(x)+m(1-t) f(y)-C m t(1-t)|y-x|^{2},
$$

holds $\forall x, y \in[0, b], b>0$ and $t, m \in[0,1]$.

The Hadamard inequality is another way of representing convex function stated in the upcoming theorem.

Theorem 1.1. [2] Let $f: I \rightarrow \mathbb{R}$ be a convex function on interval $I \subset \mathbb{R}$ and $x, y \in I$ where $x<y$. Then the following inequality holds:

$$
f\left(\frac{x+y}{2}\right) \leq \frac{1}{y-x} \int_{x}^{y} f(u) d u \leq \frac{f(x)+f(y)}{2} .
$$

If order in inequality (1.6) is reversed, then it holds for concave function.

The Riemann-Liouville fractional integrals are defined as follows:

Definition 1.5. [10] Let $f \in L_{1}[x, y]$. The Riemann-Liouville fractional integral operators of order $\beta>0$ are defined as follows:

$$
\begin{aligned}
& J_{x^{+}}^{\beta} f(u)=\frac{1}{\Gamma(\beta)} \int_{x}^{u}(u-t)^{\beta-1} f(t) d t, u>x, \\
& J_{y^{-}}^{\beta} f(u)=\frac{1}{\Gamma(\beta)} \int_{u}^{y}(t-u)^{\beta-1} f(t) d t, u<y .
\end{aligned}
$$

The following version of the Hadamard inequality for convex functions via Riemann-Liouville fractional integrals was proved by Sarikaya et al. in [9]: 
Theorem 1.2. [9] Let $f:[x, y] \rightarrow \mathbb{R}$ be a positive function with $0 \leq x<y$ and $f \in L_{1}[x, y]$. If $f$ is convex function on $[x, y]$, then the following inequality for fractional integrals holds:

$$
f\left(\frac{x+y}{2}\right) \leq \frac{\Gamma(\beta+1)}{2(y-x)^{\beta}}\left[\left(J_{x^{+}}^{\beta} f\right)(y)+\left(J_{y^{-}}^{\beta} f\right)(x)\right] \leq \frac{f(x)+f(y)}{2}
$$

with $\beta>0$.

In [9], they also studied the error estimations of this fractional Hadamard inequality by establishing an identity. Another version of the Hadamard inequality was proved by Sarikaya and Yildirim in [11].

Theorem 1.3. [11] Let $f:[x, y] \rightarrow \mathbb{R}$ be a positive function with $0 \leq x<y$. If $f \in L_{1}[x, y]$, then the following fractional integrals equality holds:

$$
f\left(\frac{x+y}{2}\right) \leq \frac{2^{\beta-1} \Gamma(\beta+1)}{(y-x)^{\beta}}\left[J_{\left(\frac{x+y}{2}\right)^{+}}^{\beta} f(y)+J_{\left(\frac{x+y}{2}\right)^{-}}^{\beta} f(x)\right] \leq \frac{f(x)+f(y)}{2} .
$$

Our aim in this paper is to study all of the above inequalities and their error estimations by applying definition of strongly $m$-convex functions.

In the upcoming section we will prove the version of Hadamard inequality for strongly $m$-convex functions, which simultaneously will represent refinement as well as generalization of Theorem 1.2. Another version of the Hadamard inequality will be proved which will provide refinement and generalization of Theorem 1.3 at the same time. Also error estimations of the fractional Hadamard inequality are given in refined form.

\section{Main Results}

Theorem 2.1. Let $f \in L_{1}[x, y]$ be a positive function with $0 \leq x<$ my. If $f$ is strongly m-convex function on $[x, m y], m \neq 0$ with modulus $C$, then the following fractional integral inequality holds:

$$
\begin{aligned}
& f\left(\frac{x+m y}{2}\right)+\frac{C m \beta}{4}\left(\frac{(x-y)^{2}}{\beta+2}+\frac{2\left(m y-\frac{x}{m}\right)^{2}}{\beta(\beta+1)(\beta+2)}+\frac{2(x-y)\left(m y-\frac{x}{m}\right)}{(\beta+1)(\beta+2)}\right) \\
& \leq \frac{\Gamma(\beta+1)}{2(m y-x)^{\beta}}\left[J_{x^{+}}^{\beta} f(m y)+m^{\beta+1} J_{y^{-}}^{\beta} f\left(\frac{x}{m}\right)\right] \\
& \leq\left(f(x)-m^{2} f\left(\frac{x}{m^{2}}\right)\right) \frac{\beta}{2(\beta+1)}+\frac{m}{2}\left(f(y)+m f\left(\frac{x}{m^{2}}\right)\right) \\
& -\frac{C m \beta\left((y-x)^{2}+m\left(y-\frac{x}{m^{2}}\right)^{2}\right)}{2(\beta+1)(\beta+2)},
\end{aligned}
$$

with $\beta, C>0$.

Proof. Since the function $f$ is strongly $m$-convex function, for $u, v \in[x, y]$ we have

$$
f\left(\frac{u+m v}{2}\right) \leq \frac{f(u)+m f(v)}{2}-\frac{C m}{4}|u-v|^{2} .
$$


By setting $u=x t+m(1-t) y$ and $v=y t+(1-t) \frac{x}{m}$, we have

$$
\begin{aligned}
& f\left(\frac{x+m y}{2}\right) \leq \frac{1}{2} f(x t+m(1-t) y)+\frac{m}{2} f\left(y t+(1-t) \frac{x}{m}\right) \\
& -\frac{C m}{4}\left|t(x-y)+(1-t)\left(m y-\frac{x}{m}\right)\right|^{2} .
\end{aligned}
$$

By multiplying inequality (2.3) with $t^{\beta-1}$ on both sides and then integrating over the interval $[0,1]$, we get

$$
\begin{aligned}
& f\left(\frac{x+m y}{2}\right) \int_{0}^{1} t^{\beta-1} d t \leq \frac{1}{2} \int_{0}^{1} f(x t+m(1-t) y) t^{\beta-1} d t \\
& +\frac{m}{2} \int_{0}^{1} f\left(y t+(1-t) \frac{x}{m}\right) t^{\beta-1} d t-\frac{C m}{4} \int_{0}^{1}\left|t(x-y)+(1-t)\left(m y-\frac{x}{m}\right)\right|^{2} t^{\beta-1} d t
\end{aligned}
$$

By change of variables we will get

$$
\begin{aligned}
& \frac{1}{\beta} f\left(\frac{x+m y}{2}\right) \\
& \leq \frac{\Gamma(\beta)}{2(m y-x)^{\beta}}\left[\frac{1}{\Gamma(\beta)} \int_{x}^{m y}(m y-u)^{\beta-1} f(u) d u+\frac{m^{\beta+1}}{\Gamma(\beta)} \int_{\frac{x}{m}}^{y}\left(v-\frac{x}{m}\right)^{\alpha-1} f(v) d v\right] \\
& -\frac{C m}{4}\left(\frac{(x-y)^{2}}{\beta+2}+\frac{2\left(m y-\frac{x}{m}\right)^{2}}{\beta(\beta+1)(\beta+2)}+\frac{2(x-y)\left(m y-\frac{x}{m}\right)}{(\beta+1)(\beta+2)}\right) .
\end{aligned}
$$

Therefore, the above inequality takes the following form:

$$
\begin{aligned}
& f\left(\frac{x+m y}{2}\right) \leq \frac{\Gamma(\beta+1)}{2(m y-x)^{\beta}}\left[J_{x^{+}}^{\beta} f(m y)+m^{\beta+1} \int_{y^{-}}^{\beta} f\left(\frac{x}{m}\right)\right] \\
& -\frac{C m \beta}{4}\left(\frac{(x-y)^{2}}{\beta+2}+\frac{2\left(m y-\frac{x}{m}\right)^{2}}{\beta(\beta+1)(\beta+2)}+\frac{2(x-y)\left(m y-\frac{x}{m}\right)}{(\beta+1)(\beta+2)}\right) .
\end{aligned}
$$

From the definition of strongly $m$-convex function with modulus $C$, for $t \in[0,1]$ we have the following inequality:

$$
\begin{aligned}
& f(t x+m(1-t) y)+m f\left(y t+(1-t) \frac{x}{m}\right) \\
& \leq t\left(f(x)-m^{2} f\left(\frac{x}{m^{2}}\right)\right)+m\left(f(y)+m f\left(\frac{x}{m^{2}}\right)\right) \\
& -C m t(1-t)\left((y-x)^{2}+m\left(y-\frac{x}{m^{2}}\right)^{2}\right) .
\end{aligned}
$$

By multiplying inequality (2.7) with $t^{\beta-1}$ on both sides and then integrating over the interval $[0,1]$, we get

$$
\begin{aligned}
& \int_{0}^{1} f(t x+m(1-t) y) t^{\beta-1} d t+m \int_{0}^{1} f\left(y t+(1-t) \frac{x}{m}\right) t^{\beta-1} d t \\
& \leq\left(f(x)-m^{2} f\left(\frac{x}{m^{2}}\right)\right) \int_{0}^{1} t^{\beta} d t+m\left(f(y)+m f\left(\frac{x}{m^{2}}\right)\right) \int_{0}^{1} t^{\beta-1} d t \\
& -C m\left((y-x)^{2}+m\left(y-\frac{x}{m^{2}}\right)^{2}\right) \int_{0}^{1} t^{\beta}(1-t) d t .
\end{aligned}
$$


By change of variables we will get

$$
\begin{aligned}
& \frac{\Gamma(\beta)}{(m y-x)^{\beta}}\left[\frac{1}{\Gamma(\beta)} \int_{x}^{m y}(m y-u)^{\beta-1} f(u) d u+\frac{m^{\beta+1}}{\Gamma(\beta)} \int_{\frac{x}{m}}^{y}\left(v-\frac{x}{m}\right)^{\beta-1} f(v) d v\right] \\
& \leq\left(f(x)-m^{2} f\left(\frac{x}{m^{2}}\right)\right) \frac{1}{(\beta+1)}+\frac{m}{\beta}\left(f(y)+m f\left(\frac{x}{m^{2}}\right)\right) \\
& -\frac{C m\left((y-x)^{2}+m\left(y-\frac{x}{m^{2}}\right)^{2}\right)}{(\beta+1)(\beta+2)} .
\end{aligned}
$$

Therefore, the above inequality takes the following form:

$$
\begin{aligned}
& \frac{\Gamma(\beta+1)}{2(m y-x)^{\beta}}\left[J_{x^{+}}^{\beta} f(m y)+m^{\beta+1} J_{y^{-}}^{\beta} f\left(\frac{x}{m}\right)\right] \\
& \leq\left(f(x)-m^{2} f\left(\frac{x}{m^{2}}\right)\right) \frac{\beta}{2(\beta+1)}+\frac{m}{2}\left(f(y)+m f\left(\frac{x}{m^{2}}\right)\right) \\
& -\frac{C m \beta\left((y-x)^{2}+m\left(y-\frac{x}{m^{2}}\right)^{2}\right)}{2(\beta+1)(\beta+2)} .
\end{aligned}
$$

From inequalities (2.6) and (2.10), one can get inequality (2.1).

Remark 2.1. (i) For $C=0$ in inequality (2.1), we get [4, Theorem 2.1], for $C \neq 0$ (2.1) is the refinement.

(ii) For $m=1$ and $C=0$ in inequality (2.1), we get Theorem 1.2.

(iii) For $m=1$ in inequality (2.1) we get the Hadamard inequality for strongly convex function.

(iv) For $\beta=1$ and $m=1$ in (2.1) we will get the refinement of the Hadamard inequality.

The next result is the refinement of another version of the Hadamard inequality for Riemann-Liouville fractional integrals stated in Theorem 1.3.

Theorem 2.2. Let $f \in L_{1}[x, y]$ be a positive function with $0 \leq x<m y$. If $f$ is a strongly m-convex function on $[x, m y], m \neq 0$ with modulus $C$, then the following fractional integral inequality holds:

$$
\begin{aligned}
& f\left(\frac{x+m y}{2}\right)+\frac{C m \beta}{4}\left[\frac{(x-y)^{2}}{4(\beta+2)}+\frac{\left(m y-\frac{x}{m}\right)^{2}\left(\beta^{2}+5 \beta+8\right)}{4 \beta(\beta+1)(\beta+2)}+\frac{(x-y)\left(m y-\frac{x}{m}\right)(\beta+3)}{2(\beta+1)(\beta+2)}\right] \\
& \leq \frac{2^{\beta-1} \Gamma(\beta+1)}{(m y-x)^{\beta}}\left[\left(J_{\left(\frac{x+m y}{2}\right)^{+}}^{\beta} f\right)(y m)+m^{\beta+1}\left(J_{\left(\frac{x+y m}{2 m}\right)^{-}}^{\beta} f\right)\left(\frac{x}{m}\right)\right] \\
& \leq \frac{\beta\left(f(x)-m^{2} f\left(\frac{x}{m^{2}}\right)\right)}{4(\beta+1)}+\frac{m}{2}\left(f(y)+m f\left(\frac{x}{m^{2}}\right)\right)-\frac{C m \beta\left((y-x)^{2}+m\left(y-\frac{x}{m^{2}}\right)^{2}\right)(\beta+3)}{8(\beta+1)(\beta+2)},
\end{aligned}
$$

with $\beta, C>0$.

Proof. For $t \in[0,1]$ and strongly $m$-convexity of function, let $u=x \frac{t}{2}+m\left(\frac{2-t}{2}\right) y$ and $v=\left(\frac{2-t}{2}\right) \frac{x}{m}+y \frac{t}{2}$ in inequality (2.2), we have 


$$
\begin{aligned}
& f\left(\frac{x+m y}{2}\right) \leq \frac{1}{2} f\left(x \frac{t}{2}+m\left(\frac{2-t}{2}\right) y\right)+\frac{m}{2} f\left(\left(\frac{2-t}{2}\right) \frac{x}{m}+y \frac{t}{2}\right) \\
& -\frac{C m}{4}\left|\frac{t}{2}(x-y)+\frac{2-t}{2}\left(m y-\frac{x}{m}\right)\right|^{2} .
\end{aligned}
$$

By multiplying (2.12) with $t^{\beta-1}$ on both sides and making integration over $[0,1]$ we get

$$
\begin{aligned}
& f\left(\frac{x+m y}{2}\right) \int_{0}^{1} t^{\beta-1} d t \leq \frac{1}{2} \int_{0}^{1} f\left(x \frac{t}{2}+m\left(\frac{2-t}{2}\right) y\right) t^{\beta-1} d t \\
& +\frac{m}{2} \int_{0}^{1} f\left(\left(\frac{2-t}{2}\right) \frac{x}{m}+y \frac{t}{2}\right) t^{\beta-1} d t \\
& -\frac{C m}{4} \int_{0}^{1}\left|\frac{t}{2}(x-y)+\frac{2-t}{2}\left(m y-\frac{x}{m}\right)\right|^{2} t^{\beta-1} d t
\end{aligned}
$$

By using change of variables and computing the last integral, from (2.13) we get

$$
\begin{aligned}
& \frac{1}{\beta} f\left(\frac{x+m y}{2}\right) \\
& \leq \frac{2^{\beta-1} \Gamma(\beta)}{(m y-x)^{\beta}}\left[\frac{1}{\Gamma(\beta)} \int_{\frac{x+m y}{2}}^{m y}(m y-u)^{\beta-1} f(u) d u+\frac{m^{\beta+1}}{\Gamma(\beta)} \int_{\frac{x}{m}}^{\frac{y m+x}{2 m}}\left(v-\frac{x}{m}\right)^{\beta-1} f(v) d v\right] \\
& -\frac{C m}{4}\left[\frac{(x-y)^{2}}{4(\beta+2)}+\frac{\left(m y-\frac{x}{m}\right)^{2}\left(\beta^{2}+5 \beta+8\right)}{4 \beta(\beta+1)(\beta+2)}+\frac{(x-y)\left(m y-\frac{x}{m}\right)(\beta+3)}{2(\beta+1)(\beta+2)}\right]
\end{aligned}
$$

Further it takes the following form

$$
\begin{aligned}
& f\left(\frac{x+m y}{2}\right) \leq \frac{2^{\beta-1} \Gamma(\beta+1)}{(m y-x)^{\beta}}\left[\left(J_{\left(\frac{x+m y}{2}\right)^{+}}^{\beta} f\right)(y m)+m^{\beta+1}\left(J_{\left(\frac{y m+x}{2 m}\right)^{-}}^{\beta} f\right)\left(\frac{x}{m}\right)\right] \\
& -\frac{C m \beta}{4}\left[\frac{(x-y)^{2}}{4(\beta+2)}+\frac{\left(m y-\frac{x}{m}\right)^{2}\left(\beta^{2}+5 \beta+8\right)}{4 \beta(\beta+1)(\beta+2)}+\frac{(x-y)\left(m y-\frac{x}{m}\right)(\beta+3)}{2(\beta+1)(\beta+2)}\right] .
\end{aligned}
$$

The first inequality of (2.11) can be seen in (2.15). Now we prove the second inequality of (2.11). Since $f$ is strongly $m$-convex function and $t \in[0,1]$, we have the following inequality:

$$
\begin{aligned}
& f\left(x \frac{t}{2}+m\left(\frac{2-t}{2}\right) y\right)+m f\left(\left(\frac{2-t}{2}\right) \frac{x}{m}+y \frac{t}{2}\right) \leq \frac{t}{2}\left(f(x)-m^{2} f\left(\frac{x}{m^{2}}\right)\right) \\
& +m\left(f(y)+m f\left(\frac{x}{m^{2}}\right)\right)-\frac{C m t(2-t)}{4}\left[(y-x)^{2}+m\left(y-\frac{x}{m^{2}}\right)^{2}\right] .
\end{aligned}
$$


By multiplying inequality (2.16) with $t^{\beta-1}$ on both sides and making integration over $[0,1]$ we get

$$
\begin{aligned}
& \int_{0}^{1} f\left(x \frac{t}{2}+m\left(\frac{2-t}{2}\right) y\right) t^{\beta-1} d t+m \int_{0}^{1} f\left(\left(\frac{2-t}{2}\right) \frac{x}{m}+y \frac{t}{2}\right) t^{\beta-1} d t \\
& \leq \frac{1}{2}\left(f(x)-m^{2} f\left(\frac{x}{m^{2}}\right)\right) \int_{0}^{1} t^{\beta} d t+m\left(f(y)+m f\left(\frac{x}{m^{2}}\right)\right) \int_{0}^{1} t^{\beta-1} d t \\
& -\frac{C m}{4}\left[(y-x)^{2}+m\left(y-\frac{x}{m^{2}}\right)\right] \int_{0}^{1} t^{\beta}(2-t) d t .
\end{aligned}
$$

By using change of variables and computing the last integral, from (2.17) we get

$$
\begin{aligned}
& \frac{2^{\beta} \Gamma(\beta)}{(m y-x)^{\beta}}\left[\frac{1}{\Gamma(\beta)} \int_{\frac{x+m y}{2}}^{m y}(m y-u)^{\beta-1} f(u) d u+\frac{m^{\beta+1}}{\Gamma(\beta)} \int_{\frac{x}{m}}^{\frac{m y+x}{2 m}}\left(v-\frac{x}{m}\right)^{\beta-1} f(v) d v\right] \\
& \leq \frac{\left(f(x)-m^{2} f\left(\frac{x}{m^{2}}\right)\right)}{2(\beta+1)}+\frac{m\left(f(y)+m f\left(\frac{x}{m^{2}}\right)\right)}{\beta}-\frac{C m\left((y-x)^{2}+m\left(y-\frac{x}{m^{2}}\right)^{2}\right)(\beta+3)}{4(\beta+1)(\beta+2)} .
\end{aligned}
$$

Further it takes the following form

$$
\begin{aligned}
& \frac{2^{\beta-1} \Gamma(\beta+1)}{(m y-x)^{\beta}}\left[\left(J_{\left(\frac{x+m y}{2}\right)^{+}}^{\beta} f\right)(y m)+m^{\beta+1}\left(J_{\left(\frac{x+y m}{2 m}\right)^{-}}^{\beta} f\right)\left(\frac{x}{m}\right)\right] \\
& \leq \frac{\beta\left(f(x)-m^{2} f\left(\frac{x}{m^{2}}\right)\right)}{4(\beta+1)}+\frac{m}{2}\left(f(y)+m f\left(\frac{x}{m^{2}}\right)\right) \\
& -\frac{C m \beta\left((y-x)^{2}+m\left(y-\frac{x}{m^{2}}\right)^{2}\right)(\beta+3)}{8(\beta+1)(\beta+2)} .
\end{aligned}
$$

From inequalities (2.15) and (2.19), we have inequality (2.11).

Remark 2.2. (i) For $C=0$ in (2.1), we will get [3, Theorem 2.1], and for $C \neq 0$ its refinement is obtained.

(ii) For $\beta=1$ and $m=1$ in (2.1) we will get refinement of the Hadamard inequality.

(iii) For $m=1$ and $C=0$ in (2.1), we will get Theorem 1.3.

(iv) For $m=1$ in inequality (2.11) we will get the Hadamard inequality for strongly convex function.

(v) For $m=1, C=0$ and $\beta=1$ in inequality (2.11), we get the inequality (1.6).

\section{Error Estimations}

Lemma 3.1. [9] Let $f:[x, y] \rightarrow \mathbb{R}$ be a differentiable mapping on $(x, y)$ with $x<y$. If $f^{\prime} \in[x, y]$, then the following fractional integrals equality holds:

$$
\begin{aligned}
& \frac{f(x)+f(y)}{2}-\frac{\Gamma(\beta+1)}{2(y-x)^{\beta}}\left[\left(J_{x^{+}}^{\beta} f\right)(y)+\left(J_{y^{-}}^{\beta} f\right)(x)\right] \\
& =\frac{y-x}{2} \int_{0}^{1}\left[(1-t)^{\beta}-t^{\beta}\right] f^{\prime}(t x+(1-t) y) d t .
\end{aligned}
$$

Theorem 3.1. Let $f:[x, y] \rightarrow \mathbb{R}$ be a differentiable mapping on $(x, y)$ with $x<m y$. If $\left|f^{\prime}\right|$ is a strongly $m$-convex function on $[x, m y], m \neq 0$, with modulus $C$, then the following fractional integrals 
inequality holds:

$$
\begin{aligned}
& \left|\frac{f(x)+f(y)}{2}-\frac{\Gamma(\beta+1)}{2(y-x)^{\beta}}\left[\left(J_{x^{+}}^{\beta} f\right)(y)+\left(J_{y^{-}}^{\beta} f\right)(x)\right]\right| \\
& \leq \frac{y-x}{2}\left[\frac{1}{(\beta+1)}\left(1-\frac{1}{2^{\beta}}\right)\left[\left|f^{\prime}(x)\right|+m\left|f^{\prime}\left(\frac{y}{m}\right)\right|\right]-\frac{2 C m\left(\frac{y}{m}-x\right)^{2}}{(\beta+2)(\beta+3)}\left(1-\frac{\beta+4}{2^{\beta+2}}\right)\right],
\end{aligned}
$$

with $\beta, C>0$.

Proof. Since $\left|f^{\prime}\right|$ is strongly $m$-convex function on $[x, m y]$ and $t \in[0,1]$, we have

$$
\begin{aligned}
& \left|f^{\prime}(t x+(1-t) y)\right| d t=\left|f^{\prime}\left(t x+m(1-t) \frac{y}{m}\right)\right| d t \\
& \leq t\left|f^{\prime}(x)\right|+m(1-t)\left|f^{\prime}\left(\frac{y}{m}\right)\right|-C m t(1-t)\left(\frac{y}{m}-x\right)^{2} .
\end{aligned}
$$

By using Lemma 3.1 and (3.3) we have

$$
\begin{aligned}
& \left|\frac{f(x)+f(y)}{2}-\frac{\Gamma(\beta+1)}{2(y-x)^{\beta}}\left[\left(J_{x^{+}}^{\beta} f\right)(y)+\left(J_{y^{-}}^{\beta} f\right)(x)\right]\right| \\
& \leq \frac{y-x}{2} \int_{0}^{1}\left|(1-t)^{\beta}-t^{\beta}\right|\left|f^{\prime}\left(t x+m(1-t) \frac{y}{m}\right)\right| d t \leq \frac{y-x}{2} \int_{0}^{1}\left|(1-t)^{\beta}-t^{\beta}\right| \\
& \left(t\left|f^{\prime}(x)\right|+m(1-t)\left|f^{\prime}\left(\frac{y}{m}\right)\right|-C m t(1-t)\left(\frac{y}{m}-x\right)^{2}\right) d t \leq \frac{y-x}{2} \\
& {\left[\int_{0}^{1 / 2}\left((1-t)^{\beta}-t^{\beta}\right)\left(t\left|f^{\prime}(x)\right|+m(1-t)\left|f^{\prime}\left(\frac{y}{m}\right)\right|-C m t(1-t)\left(\frac{y}{m}-x\right)^{2}\right) d t\right.} \\
& \left.+\int_{1 / 2}^{1}\left(t^{\beta}-(1-t)^{\beta}\right)\left(t\left|f^{\prime}(x)\right|+m(1-t)\left|f^{\prime}\left(\frac{y}{m}\right)\right|-C m t(1-t)\left(\frac{y}{m}-x\right)^{2}\right) d t\right] \\
& \leq \frac{y-x}{2}\left[\left|f^{\prime}(x)\right|\left(\frac{1}{(\beta+1)(\beta+2)}-\frac{(1 / 2)^{\beta+1}}{\beta+1}\right)+m\left|f^{\prime}\left(\frac{y}{m}\right)\right|\left(\frac{1}{(\beta+2)}-\frac{(1 / 2)^{\beta+1}}{\beta+1}\right)\right. \\
& -\frac{C m\left(\frac{y}{m}-x\right)^{2}}{(\beta+2)(\beta+3)}\left(1-\frac{\beta+4}{2^{\beta+2}}\right)+\left|f^{\prime}(x)\right|\left(\frac{1}{\beta+2}-\frac{(1 / 2)^{\beta+1}}{\beta+1}\right) \\
& \left.+m\left|f^{\prime}\left(\frac{y}{m}\right)\right|\left(\frac{1}{(\beta+1)(\beta+2)}-\frac{(1 / 2)^{\beta+1}}{\beta+1}\right)-\frac{C m\left(\frac{y}{m}-x\right)^{2}}{(\beta+2)(\beta+3)}\left(1-\frac{\beta+4}{2^{\beta+2}}\right)\right] .
\end{aligned}
$$

After simplify the last inequality of (3.4), we get the inequality (3.2).

Remark 3.1. (i) If $m=1, C=0$ in inequality (3.2), we get [9, Theorem 3], for $C>0$ we get its refinement.

(ii) If $m=1, C=0$ and $\beta=1$ in inequality (3.2), we get [1, Theorem 2.2], for $C>0$ we get its refinement.

Corollary 3.1. For $\beta=1$

$$
\left|\frac{f(x)+f(y)}{2}-\frac{1}{(y-x)} \int_{x}^{y} f(u) d u\right| \leq \frac{y-x}{8}\left[\left|f^{\prime}(x)\right|+m\left|f^{\prime}\left(\frac{y}{m}\right)\right|\right]-\frac{C m\left(\frac{y}{m}-x\right)^{3}}{32},
$$

with $C>0$. 
Theorem 3.2. Let $f:[x, y] \rightarrow \mathbb{R}$ be a differentiable mapping on $(x, y)$ with $x<m y$. If $\left|f^{\prime}\right|^{q}$ is strongly $m$-convex on $[x, y]$ for $q \geq 1$, then the following inequality for fractional integrals holds:

$$
\begin{aligned}
& \mid \frac{2^{\beta-1} \Gamma(\beta+1)}{(m y-x)^{\beta}}\left[\left(J_{\left(\frac{x+m y}{2}\right)^{+}}^{\beta} f\right)(m y)+m^{\beta+1}\left(J_{\left(\frac{x+m y}{2 m}\right)^{-}}^{\beta} f\right)\left(\frac{x}{m}\right)\right] \\
& -\frac{1}{2}\left[f\left(\frac{x+m y}{2}\right)+m f\left(\frac{x+m y}{2 m}\right)\right] \mid \leq \frac{m y-x}{4(\beta+1)}\left(\frac{1}{2(\beta+2)}\right)^{\frac{1}{q}} \\
& {\left[\left(\left|f^{\prime}(x)\right|^{q}(\beta+1)+m\left|f^{\prime}(y)\right|^{q}(\beta+3)-\frac{C m(y-x)^{2}(\beta+1)(\beta+4)}{2(\beta+3)}\right)^{\frac{1}{q}}\right.} \\
& \left.+\left(\left|f^{\prime}(y)\right|^{q}(\beta+1)+m(\beta+3)\left|f^{\prime}\left(\frac{x}{m^{2}}\right)\right|^{q}-\frac{C m\left(\frac{x}{m^{2}}-y\right)^{2}(\beta+1)(\beta+4)}{2(\beta+3)}\right)^{\frac{1}{q}}\right],
\end{aligned}
$$

with $\beta>0$.

Proof. By applying [3, Lemma 2.3] and strong $m$-convexity of $\left|f^{\prime}\right|$, let $q=1$ we have

$$
\begin{aligned}
& \mid \frac{2^{\beta-1} \Gamma(\beta+1)}{(m y-x)^{\beta}}\left[\left(J_{\left(\frac{x+m y}{2}\right)^{+}}^{\beta} f\right)(m y)+m^{\beta+1}\left(J_{\left(\frac{x+m y}{2 m}\right)^{-}}^{\beta} f\right)\left(\frac{x}{m}\right)\right] \\
& -\frac{1}{2}\left[f\left(\frac{x+m y}{2}\right)+m f\left(\frac{x+m y}{2 m}\right)\right] \mid \\
& \leq \frac{m y-x}{4}\left[\int_{0}^{1}\left|t^{\beta} f^{\prime}\left(\frac{t}{2} x+m\left(\frac{2-t}{2}\right) y\right)\right| d t+\int_{0}^{1}\left|t^{\beta} f^{\prime}\left(\left(\frac{2-t}{2}\right) \frac{x}{m}+\frac{t}{2} y\right)\right| d t\right] \\
& \leq \frac{m y-x}{4}\left[\left(\frac{\left|f^{\prime}(x)\right|-m\left|f^{\prime}(y)\right|+\left|f^{\prime}(y)\right|-m\left|f^{\prime}\left(\frac{x}{m^{2}}\right)\right|}{2}\right) \int_{0}^{1} t^{\beta+1} d t+m\right. \\
& \left.\left(\left|f^{\prime}(y)\right|+\left|f^{\prime}\left(\frac{x}{m^{2}}\right)\right|\right) \int_{0}^{1} t^{\beta} d t-\frac{C m}{4}\left((y-x)^{2}+m\left(\frac{x}{m^{2}}-y\right)^{2}\right) \int_{0}^{1} t^{\beta+1}(2-t) d t\right] \\
& =\frac{m y-x}{4}\left[\left(\frac{\left|f^{\prime}(x)\right|-m\left|f^{\prime}(y)\right|+\left|f^{\prime}(y)\right|-m\left|f^{\prime}\left(\frac{x}{m^{2}}\right)\right|}{2(\beta+2)}\right)+\frac{m\left(\left|f^{\prime}(y)\right|+\left|f^{\prime}\left(\frac{x}{m^{2}}\right)\right|\right)}{\beta+1}\right. \\
& \left.-\frac{C m(\beta+4)}{4(\beta+2)(\beta+3)}\left((y-x)^{2}+m\left(\frac{x}{m^{2}}-y\right)^{2}\right)\right] .
\end{aligned}
$$

Now, for strongly $m$-convexity of $\left|f^{\prime}\right|^{q}, q>1$, using power mean inequality we get

$$
\begin{aligned}
& \mid \frac{2^{\beta-1} \Gamma(\beta+1)}{(m y-x)^{\beta}}\left[\left(J_{\left(\frac{x+m y}{2}\right)^{+}}^{\beta} f\right)(m y)+m^{\beta+1}\left(J_{\left(\frac{x+m y}{2 m}\right)^{-}}^{\beta} f\right)\left(\frac{x}{m}\right)\right]-\frac{1}{2}\left[f\left(\frac{x+m y}{2}\right)\right. \\
& \left.+m f\left(\frac{x+m y}{2 m}\right)\right] \mid \leq \frac{m y-x}{4}\left(\int_{0}^{1} t^{\beta} d t\right)^{1-\frac{1}{q}}\left[\left(\int_{0}^{1} t^{\beta}\left|f^{\prime}\left(\frac{t}{2} x+m\left(\frac{2-t}{2}\right) y\right)\right|^{q} d t\right)^{\frac{1}{q}}\right. \\
& \left.+\left(\int_{0}^{1} t^{\beta}\left|f^{\prime}\left(\left(\frac{2-t}{2}\right) \frac{x}{m}+\frac{t}{2} y\right)\right|^{q} d t\right)^{\frac{1}{q}}\right] \leq \frac{m y-x}{4(\beta+1)^{\frac{1}{p}}}\left(\frac{\left|f^{\prime}(x)\right|^{q}-m\left|f^{\prime}(y)\right|^{q}}{2} \int_{0}^{1} t^{\beta+1} d t\right.
\end{aligned}
$$




$$
\begin{aligned}
& \left.+m\left|f^{\prime}(y)\right|^{q} \int_{0}^{1} t^{\beta} d t-\frac{C m(y-x)^{2}}{4} \int_{0}^{1} t^{\beta+1}(2-t) d t\right)^{\frac{1}{q}}+\left(\frac{\left|f^{\prime}(y)\right|^{q}-m\left|f^{\prime}\left(\frac{x}{m^{2}}\right)\right|^{q}}{2}\right. \\
& \left.\int_{0}^{1} t^{\beta+1} d t+m\left|f^{\prime}\left(\frac{x}{m^{2}}\right)\right|^{q} \int_{0}^{1} t^{\beta} d t-\frac{C m\left(\frac{x}{m^{2}}-y\right)^{2}}{4} \int_{0}^{1} t^{\beta+1}(2-t) d t\right)^{\frac{1}{q}} \leq \frac{m y-x}{4(\beta+1)^{\frac{1}{p}}} \\
& {\left[\left(\frac{\left|f^{\prime}(x)\right|^{q}}{2(\beta+2)}+m \frac{\left|f^{\prime}(y)\right|^{q}(\beta+3)}{2(\beta+1)(\beta+2)}-\frac{C m(y-x)^{2}(\beta+4)}{4(\beta+2)(\beta+3)}\right)^{\frac{1}{q}}+\left(\frac{\left|f^{\prime}(y)\right|^{q}}{2(\beta+2)}\right.\right.} \\
& \left.\left.+m \frac{\left|f^{\prime}\left(\frac{x}{m^{2}}\right)\right|^{q}(\beta+3)}{2(\beta+1)(\beta+2)}-\frac{C m\left(\frac{x}{m^{2}}-y\right)^{2}(\beta+4)}{4(\beta+2)(\beta+3)}\right)^{\frac{1}{q}}\right]=\frac{m y-x}{4(\beta+1)}\left(\frac{1}{2(\beta+2)}\right)^{\frac{1}{q}} \\
& {\left[\left(\left|f^{\prime}(x)\right|^{q}(\beta+1)+m\left|f^{\prime}(y)\right|^{q}(\beta+3)-\frac{C m(y-x)^{2}(\beta+4)(\beta+1)}{2(\beta+3)}\right)^{\frac{1}{q}}\right.} \\
& \left.+\left(\left|f^{\prime}(y)\right|^{q}(\beta+1)+m\left|f^{\prime}\left(\frac{x}{m^{2}}\right)\right|^{q}(\beta+3)-\frac{C m\left(\frac{x}{m^{2}}-y\right)^{2}(\beta+4)(\beta+1)}{2(\beta+3)}\right)^{\frac{1}{q}}\right] .
\end{aligned}
$$

So, we have inequality 3.5 .

Remark 3.2. (i) If $C=0$ in in inequality (3.5), we have [3, Theorem 2.4].

(ii) If $m=1$ and $C=0$ in inequality (3.5), we have [11, Theorem 5].

(iii) If $m=1, C=0$ and $\beta=1$ in inequality 3.5 we get the inequality which is proved by Kirmaci [5].

Corollary 3.2. If $m=1, q=1$ and $\beta=1$ in 3.5, we get

$$
\left|\frac{1}{y-x} \int_{x}^{y} f(u) d u-f\left(\frac{x+y}{2}\right)\right| \leq \frac{y-x}{8}\left[\left|f^{\prime}(x)\right|+\left|f^{\prime}(y)\right|-\frac{5 C(y-x)^{2}}{12}\right] .
$$

Theorem 3.3. Let $f:[x, y] \rightarrow \mathbb{R}$ be a differentiable mapping on $(x, y)$ with $x<y$. If $\left|f^{\prime}\right|^{q}$ is strongly $m$-convex function on $[x, y]$ for $q>1$, then the following fractional integrals inequality holds:

$$
\begin{aligned}
& \quad \frac{2^{\beta-1} \Gamma(\beta+1)}{(m y-x)^{\beta}}\left[\left(J_{\left(\frac{x+m y}{2}\right)^{+}}^{\beta} f\right)(m y)+m^{\beta+1}\left(J_{\left(\frac{x+m y}{2 m}\right)^{-}}^{\beta} f\right)\left(\frac{x}{m}\right)\right] \\
& -\frac{1}{2}\left[f\left(\frac{x+m y}{2}\right)+m f\left(\frac{x+m y}{2 m}\right)\right] \mid \leq \frac{m y-x}{16}\left(\frac{4}{\beta p+1}\right)^{\frac{1}{p}} \\
& {\left[\left(\left|f^{\prime}(x)\right|^{q}+3 m\left|f^{\prime}(y)\right|^{q}-\frac{2 C m(y-x)^{2}}{3}\right)^{\frac{1}{q}}+\left(3 m\left|f^{\prime}\left(\frac{x}{m^{2}}\right)\right|^{q}+\left|f^{\prime}(y)\right|^{q}\right.\right.} \\
& \left.\left.-\frac{2 C m\left(\frac{x}{m^{2}}-y\right)^{2}}{3}\right)^{\frac{1}{q}}\right] \leq \frac{m y-x}{16}\left(\frac{4}{\beta p+1}\right)^{\frac{1}{p}}\left[\left|f^{\prime}(x)\right|+\left|f^{\prime}(y)\right|\right. \\
& \left.+3 m\left(\left|f^{\prime}\left(\frac{x}{m^{2}}\right)\right|+\left|f^{\prime}(y)\right|\right)-\frac{2 C m}{3}\left((y-x)^{2}+\left(\frac{x}{m^{2}}-y\right)^{2}\right)\right]
\end{aligned}
$$

where $\frac{1}{p}+\frac{1}{q}=1, \beta>0$. 
Proof. By applying [3, Lemma 2.3], using Holder inequality and strong $m$-convexity of $\left|f^{\prime}\right|^{q}$, we get

$$
\begin{aligned}
& \mid \frac{2^{\beta-1} \Gamma(\beta+1)}{(m y-x)^{\beta}}\left[\left(J_{\left(\frac{x+m y}{2}\right)^{+}}^{\beta} f\right)(m y)+m^{\beta+1}\left(J_{\left(\frac{x+m y}{2 m}\right)^{-}}^{\beta} f\right)\left(\frac{x}{m}\right)\right] \\
& -\frac{1}{2}\left[f\left(\frac{x+m y}{2}\right)+m f\left(\frac{x+m y}{2 m}\right)\right] \mid \leq \frac{m y-x}{4}\left(\int_{0}^{1} t^{p \beta} d t\right)^{\frac{1}{p}} \\
& {\left[\left(\int_{0}^{1}\left|f^{\prime}\left(x \frac{t}{2}+m\left(\frac{(2-t)}{2}\right) y\right)\right|^{q} d t\right)^{\frac{1}{q}}+\left(\int_{0}^{1}\left|f^{\prime}\left(y \frac{t}{2}+\left(\frac{2-t}{2}\right) \frac{x}{m}\right)\right|^{q} d t\right)^{\frac{1}{q}}\right]} \\
& \leq \frac{m y-x}{4}\left(\frac{1}{\beta p+1}\right)^{\frac{1}{p}}\left[\left(\left|f^{\prime}(x)\right|^{q} \int_{0}^{1} \frac{t}{2} d t+m\left|f^{\prime}(y)\right|^{q} \int_{0}^{1} \frac{2-t}{2} d t-\frac{C m(y-x)^{2}}{4} \int_{0}^{1} t(2-t) d t\right)^{\frac{1}{q}}\right. \\
& \left.+\left(\left|f^{\prime}(y)\right|^{q} \int_{0}^{1} \frac{t}{2} d t+m\left|f^{\prime}\left(\frac{x}{m^{2}}\right)\right|^{q} \int_{0}^{1}\left(\frac{2-t}{2}\right) d t-\frac{C m\left(\frac{x}{m^{2}}-y\right)^{2}}{4} \int_{0}^{1} t(2-t) d t\right)^{\frac{1}{q}}\right] . \\
& +\frac{m y-x}{16}\left(\frac{4}{\beta p+1}\right)^{\frac{1}{p}}\left[\left(\left|f^{\prime}(x)\right|^{q}+3 m\left|f^{\prime}(y)\right|^{q}-\frac{2 C m(y-x)^{2}}{3}\right)^{\frac{1}{q}}\right. \\
& \left.+\left(3 m\left|f^{\prime}\left(\frac{x}{m^{2}}\right)\right|^{q}+\left|f^{\prime}(y)\right|^{q}-\frac{2 C m\left(\frac{x}{m^{2}}-y\right)^{2}}{3}\right)^{\frac{1}{q}}\right] \leq \frac{m y-x}{16}\left(\frac{4}{\beta p+1}\right)^{\frac{1}{p}} \\
& {\left[\left|f^{\prime}(x)\right|+\left|f^{\prime}(y)\right|+3 m\left(\left|f^{\prime}(y)\right|+\left|f^{\prime}\left(\frac{x}{m^{2}}\right)\right|\right)-\frac{2 C m}{3}\left((y-x)^{2}+\left(\frac{x}{m^{2}}-y\right)^{2}\right)\right] .}
\end{aligned}
$$

We have used $A^{q}+B^{q} \leq(A+B)^{q}$, for $A \geq 0, B \geq 0$.

Remark 3.3. (i) If $C=0$ in in 3.8, we have [3, Theorem 2.7] .

(ii) If $m=1$ and $C=0$ in 3.8, we have [11, Theorem 6].

Corollary 3.3. For $q=1$ and $\beta=1$ and $m=1$, we have

$$
\left|\frac{1}{(y-x)} \int_{x}^{y} f(u) d u-f\left(\frac{x+y}{2}\right)\right| \leq \frac{y-x}{4}\left(\frac{4}{p+1}\right)^{\frac{1}{p}}\left[\left|f^{\prime}(x)\right|+\left|f^{\prime}(y)\right|-\frac{C(y-x)^{2}}{3}\right] .
$$

Corollary 3.4. For $\beta=1$ and $m=1$, we have

$$
\begin{aligned}
& \left|\frac{1}{(y-x)} \int_{x}^{y} f(u) d u-f\left(\frac{x+y}{2}\right)\right| \leq \frac{y-x}{16}\left(\frac{4}{p+1}\right)^{\frac{1}{p}} \\
& {\left[\left(\left|f^{\prime}(a)\right|+3\left|f^{\prime}(y)\right|-\frac{2 C(y-x)^{2}}{3}\right)^{\frac{1}{q}}+\left(3\left|f^{\prime}(x)\right|+\left|f^{\prime}(y)\right|-\frac{2 C(y-x)^{2}}{3}\right)^{\frac{1}{q}}\right] .}
\end{aligned}
$$

Conflicts of Interest: The author(s) declare that there are no conflicts of interest regarding the publication of this paper.

\section{References}

[1] S.S. Dragomir, R.P. Agarwal, Two inequalities for differentiable mappings and applications to special means of real numbers and to trapezoidal formula, Appl. Math. Lett. 11 (1998), 91-95. https://doi.org/10.1016/ S0893-9659(98) 00086-X. 
[2] S.S. Dragomir, C.E.M. Pearce, Selected topics on Hermite-Hadamard inequalities and applications, RGMIA Monographs, Victoria University, 2000. http://rgmia.org/monographs/hermite_hadamard.html.

[3] G. Farid, A. Ur. Rehman, B. Tariq, On Hadamard-type inequalities for $m$-convex functions via Riemann-Liouville fractional integrals, Stud. Univ. Babeș-Bolyai Math. 62 (2017), 141-150. https://doi.org/10.24193/subbmath. 2017.2 .01$.

[4] G. Farid, A. Ur. Rehman, B. Tariq, A. Waheed, On Hadamard type inequalities for $m$-convex functions via fractional integrals, J. Inequal. Spec. Funct. 7 (2016), 150-167.

[5] U. Kirmaci, Inequalities for differentiable mappings and applications to special means of real numbers to midpoint formula, Appl. Math. Comput. 147 (2004), 137-146. https://doi.org/10.1016/S0096-3003(02)00657-4.

[6] T. Lara, N. Merentes, R. Quintero, E. Rosales, On Strongly m-convex functions, Math. Aeterna, 5 (2015), $521-535$.

[7] B.T. Polyak, Existence theorems and convergence of minimizing sequences in extremum problems with restrictions, Soviet Math. Doklady, 7 (1966), 72-75.

[8] A.W. Roberts, D.E. Varberg, Convex functions, Academic Press, New York, 1973.

[9] M. Z. Sarikaya, E. S. H. Yaldiz, N. Bașak, Hermite-Hadamard's inequalities for fractional integrals and related fractional inequalities, Math. Comput. Model. 57 (2013), 2403-2407. https://doi.org/10.1016/j.mcm.2011. 12.048.

[10] H.M. Srivastava, Z. Tomovski, Fractional calculus with an integral operator containing generalized Mittag-Leffler function in the kernel, Appl. Math. Comput. 211 (2009), 198-210. https://doi.org/10.1016/j.amc.2009.01. 055.

[11] M.Z. Sarikaya, H. Yildirim, On Hermit-Hadamard type inequalities for Rieman-Liouville fractional integrals, Miskolc Math. Notes, 17 (2017), 1049-1059.

[12] G. Toader, Some generalizations of the convexity, in: Proceedings of the Colloquium on Approximation and Optimization, Univ. Cluj-Napoca, Cluj-Napoca, (1985), 329-338. 